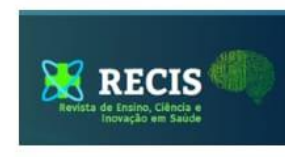

ISSN: $2675-9683$

\section{Revista de Ensino, Ciência e Inovação em Saúde}

Homepage:http://recis.huunivasf.ebserh.gov.br

\title{
Prevalência de lesões musculoesqueléticas em jovens e adultos praticantes de Crossfit $^{\circledR}$ : revisão bibliográfica
}

\section{Prevalence of musculoskeletal injuries in young and adult Crossfit practitioners: review bibliographic}

\author{
Carolina Cunha Carvalho ${ }^{1}$; Deysi Micaelli Rodrigues Cantarelli ${ }^{1}$; Allana Núbia Santos \\ Araújo'; Pedro Guilherme Campos Lima ${ }^{1}$; Danilo Sobral da Silva Fernandes²; Laís Regina \\ de Holanda ${ }^{3}$
}
${ }^{1}$ Graduandos do curso de Fisioterapia na Faculdade UniBRAS - Juazeiro/BA, ${ }^{2}$ Professor da Faculdade UniBRAS Juazeiro- Juazeiro/BA, ${ }^{3}$ Doutoranda no Programa de Pós-graduação em Reabilitação e Desempenho Funcional na Universidade de Pernambuco - Petrolina/PE.

Autor correspondente: carolina_cunhacarvalho@hotmail.com

Artigo recebido em 16/09/2021e aceito em 09/11/2021

\begin{abstract}
RESUMO
O CrossFit é uma modalidade de atividade física, de alta intensidade, que tem como objetivo principal promover condicionamento físico, melhorando as capacidades físicas do corpo através de exercícios variados, movimentos funcionais e treinamentos de alta intensidade. Apesar de apresentar exercícios com benefícios na saúde em geral, em programas de condicionamento extremo como esse, há riscos de lesões musculoesqueléticas, lesões ligamentares e até rabdomiólise. O objetivo do estudo foi realizar um levantamento bibliográfico para analisar a prevalência de lesões musculoesqueléticas em praticantes de Crossfit. Trata-se de uma revisão da literatura. A coleta foi realizada de janeiro a junho de 2021, nas bases de dados PubMed, BVS, e Scielo utilizando artigos publicados de 2017 a 2021. Embora o CrossFit possua um amplo número de participantes, na literatura os resultados positivos, que mostram seus benefícios ainda não confirmam um alto nível de evidência. Além disto, pode-se afirmar que o índice ou proporção de lesões, de acordo como mostrado pela maioria dos estudos incluídos, deve levar em consideração grande variedade de fatores . De acordo com a literatura, conclui-se que o CrossFit é ser uma modalidade segura, desde que realizada da forma adequada para cada indivíduo e com acompanhamento do profissional responsável. Porém ainda são necessários mais estudos sobre o tema, pois existem lacunas na literatura, apresentando poucas evidências de qualidade e divergência na literatura.

Palavras-chave: Treinamento Intervalado de Alta Intensidade; Prevalência; Traumatismo em atletas
\end{abstract}

\section{ABSTRACT}

CrossFit is a high-intensity physical activity modality, whose main objective is to promote physical conditioning, improving the body's physical capacities through varied exercises, functional movements and high-intensity training. Despite presenting exercises with general health benefits, in extreme conditioning programs like this one, there are risks of musculoskeletal injuries, ligament injuries and even rhabdomyolysis.

Carvalho, C. C. et al. / Revista de Ensino, Ciência e Inovação em Saúde v. 2 n. 3 (2021) p. 19-25 
The aim of the study was to conduct a bibliographic survey to analyze the prevalence of musculoskeletal injuries in Crossfit practitioners. This is a literature review. The collection was carried out from January to June 2021, in the PubMed, BVS, and Scielo databases using articles published from 2017 to 2021. Although CrossFit has a large number of participants, in the literature the positive results, which still show its benefits, do not confirm a high level of evidence. Furthermore, it can be stated that the rate or proportion of injuries, as shown by most of the included studies, must take into account a wide variety of factors. According to the literature, it is concluded that CrossFit seems to be a safe modality, as long as it is performed properly for each individual and monitored by the responsible professional. However, more studies on the subject are still needed, as there are gaps in the literature, with little evidence of quality and divergence in the literature.

Keywords: High Intensity Interval Training; Prevalence; Athletic Injuries;

\section{INTRODUÇÃO}

O CrossFit é uma modalidade esportiva, de alta intensidade que envolve atividades de força e condicionamento físico geral, esse treinamento é relativamente novo na população e que vem crescendo o interesse pela sua prática. Tem como objetivo principal promover condicionamento físico, melhorando as capacidades físicas do corpo como a resistência cardiorrespiratória, força, potência, velocidade, coordenação, flexibilidade, agilidade, equilíbrio e precisão, preparando o praticante para qualquer desafio físico e consequentemente melhorando a saúde através de exercícios variados, compostos de movimentos funcionais e treinamentos de alta intensidade. ${ }^{\mathbf{1 , 2}}$

$\mathrm{O}$ "treino do dia", conhecido como Workout of the Day (WOD), é um conjunto de exercícios alternados, compostos por: levantamento de peso olímpico, agachamentos, arrebates, arremessos, exercícios aeróbicos e movimentos de ginástica, em que a carga imposta e o movimento são ajustados individualmente de acordo com o nível de habilidade e o condicionamento de cada participante. ${ }^{2}$

Apesar do CrossFit apresentar programas de exercícios eficientes e seguros com potenciais benefícios na saúde em geral, respostas cardiovasculares e aptidão física, outros estudos evidenciam significativos riscos de lesão. Estes programas incluem exercícios que mal orientados podem ser indevidamente executados ou realizados de modo extenuante pelo aumento do volume, carga imposta e pela competição gerada durante os treinos, podendo gerar lesões musculoesqueléticas, ligamentares e até rabdomiólise. ${ }^{3,4,5}$

As lesões são caracterizadas por movimentos que impedem a realização de atividades de vida diária, como treinar e trabalhar, por qualquer período de tempo. Na prática do CrossFit, as lesões descritas na literatura são desenvolvidas por uso excessivo, por sobrecarga que pode levar a alterações crônicas nos tendões, lesões traumáticas e lesões gerais, sendo as articulações do ombro, cotovelo, região lombar e joelho as mais afetadas. ${ }^{1,2}$

Este estudo justifica-se pela necessidade de conhecer a prevalência de lesão musculoesquelética nesta modalidade esportiva, podendo contribuir para abordagens de reabilitação e prevenção nos indivíduos praticantes de CrossFit. Dessa forma o objetivo desse estudo é realizar um levantamento bibliográfico para a prevalência de lesões musculoesqueléticas em praticantes de CrossFit.

\section{MATERIAL E MÉTODOS}

O estudo trata-se de uma revisão bibliográfica de literatura. Foi realizada uma pesquisa bibliográfica nos meses de janeiro a junho de 2021, nas bases de dados United StatesNational Library of Medicine (PubMed), Biblioteca virtual em saúde (BVS), e Scientific Eletronic Library Online (Scielo) utilizando artigos publicados de 2017 a 2021.

Os termos utilizados para recrutamento dos artigos para pesquisa incluíram palavras-chave na língua portuguesa como "Lesões Esportivas" and "Treinamento Intervalado de Alta Intensidade" and "Prevalência" e na língua inglesa com os termos: "Sports injuries" and "High Intensity Interval Training" and "Prevalence".

Foram incluídos ensaios clínicos randomizados e estudos epidemiológicos que incluíam lesões musculoesqueléticas em praticantes de CrossFit jovens e adultos aparentemente saudáveis, de ambos os sexos e de idade igual ou superior a 15 anos, na língua inglesa ou portuguesa.

Foram excluídos da pesquisa estudos voltados para a população pediátrica ou para alguma população específica com patologias, ou que não tinha acesso gratuito e artigos incompletos, além de revisões sistemáticas e meta-análises. 
Revista de Ensino, Ciência e Inovação em Saúde v.2, n.3 (2021) 19-25

ISSN: 2675-9683/DOI: $10.51909 /$ recis.v2i3.166

\section{DESENVOLVIMENTO}

A pesquisa bibliográfica resultou no total de 24 artigos encontrados nas bases de dados escolhidas, sendo removidos 18 artigos por não cumprirem os critérios de inclusão do estudo. Com isso, foram incluídos seis artigos na revisão, conforme mostrado na Figura 1. As características dos 6 artigos selecionados estão descritos na Tabela 1.

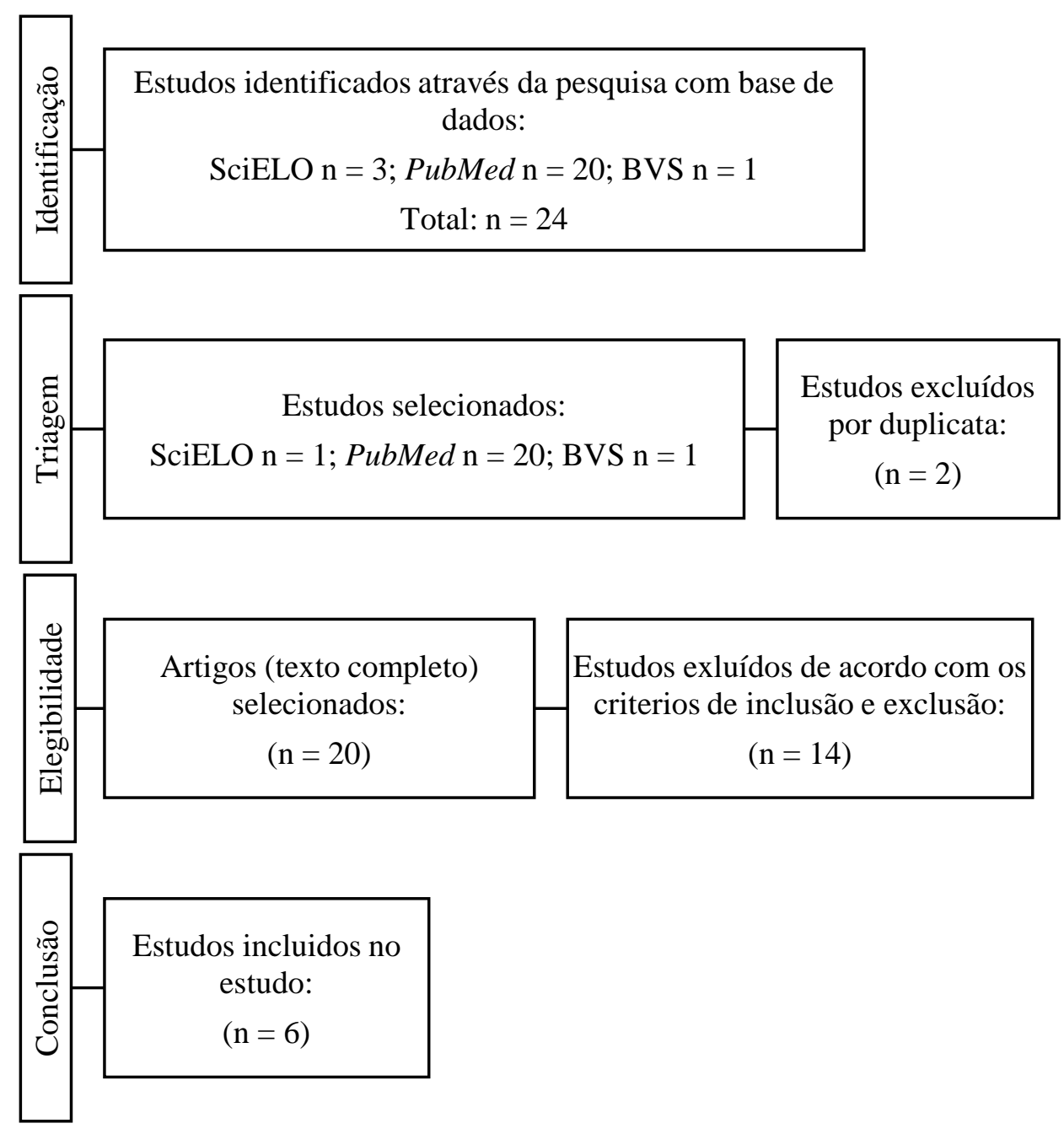

Figura 1 -Fluxograma de seleção e inclusão dos artigos no estudo 
Revista de Ensino, Ciência e Inovação em Saúde v.2, n.3 (2021) 19-25

ISSN: 2675-9683/DOI: $10.51909 /$ recis.v2i3.166

Tabela 1 - Características dos estudos selecionados

\begin{tabular}{|c|c|c|c|}
\hline $\begin{array}{c}\text { AUTOR } \\
\text { (ANO) }\end{array}$ & $\begin{array}{l}\text { TIPO DO ESTUDO } \\
\text { (AMOSTRA) }\end{array}$ & $\begin{array}{l}\text { MÉTODO DE } \\
\text { ANÁLISE }\end{array}$ & RESULTADOS \\
\hline $\begin{array}{l}\text { Costa et al } \\
\text { (2019) }\end{array}$ & $\begin{array}{l}\text { Estudo observacional, } \\
\text { descritivo, transversal e } \\
\text { epidemiológico. } \\
414 \text { praticantes de ambos } \\
\text { os sexos. }\end{array}$ & $\begin{array}{c}\text { Questionário impresso } \\
\text { aplicado diretamente } \\
\text { aos praticantes de } \\
\text { CrossFit }\end{array}$ & $\begin{array}{c}\text { O estudo mostrou que } 37,9 \% \text { dos } \\
\text { praticantes sofreram lesões durante a } \\
\text { prática do CrossFit. A taxa de lesão foi } \\
\text { de } 3,24 \text { por } 1000 \text { horas de } \\
\text { treinamento.Os locais de lesões mais } \\
\text { comuns foram o ombro, coluna lombar } \\
\text { perna. As lesões mais comuns foram } \\
\text { tensão muscular, lesão por sobrecarga, e } \\
\text { contusão. }\end{array}$ \\
\hline
\end{tabular}

$\begin{array}{ccc} & \text { Estudo epidemiológico } \\ \text { Feito et al } & \text { descritivo. } & \\ \mathbf{( 2 0 1 8 )} & \begin{array}{c}3.049 \text { participantes de } \\ \text { ambos os sexos. }\end{array} & \text { Questionário online }\end{array}$

Estudo epidemiológico descritivo.

al (2017)
Questionário eletrônico sexos.
Ombros, costas, joelhos, cotovelos e punhos foram os locais mais comuns de lesões relatados em ambos os sexos, sendo que o maior número de lesões ocorreu naqueles que tinham um alto volume de treino semanal ( 3 a 5 dias).

$56,1 \%$ dos praticantes sofreram pelo menos uma lesão ao praticar Crossfit. $\mathrm{O}$ ombro, a parte inferior das costas e joelho eram os mais comumente lesionados durante WODs. Maior parte das lesões foi originada por uso excessivo $(58,7 \%)$.

$\begin{array}{ccc}\text { Minghelli } & \text { Estudo epidemiológico e } & \\ \text { et al (2019) } & \text { transversal. } & \text { Questionário em forma de } \\ 270 \text { participantes de } & \text { Entrevista Estruturada } \\ & \text { ambos os sexos. } & \end{array}$

$29,6 \%$ dos participantes relataram ter sofrido algum tipo de lesão desde o início da prática. A taxa de lesão resultante foi de 1,34 por 1000 horas de exercícios. As regiões corporais mais acometidas por lesões foram o ombro, coluna lombar e joelho.

$26 \%$ dos entrevistados sofreram lesões nos seis meses anteriores, a incidência de lesões foi de 2,3 / 1000 horas de treinamento do atleta, sendo mais

$\begin{array}{ccc}\text { Montalvo } & \text { Estudo epidemiológico. } & \\ \text { et al (2017) } & \begin{array}{c}191 \text { participantes de } \\ \text { ambos os sexos. }\end{array}\end{array}$




\section{Szeles et al (2020)}

\begin{abstract}
Questionário impresso para recrutamento dos participantes e questionários online a cada duas semanas para acompanhamento dos mesmos
\end{abstract}

A taxa de lesão foi de 18,9 por 1000 horas de exposição CrossFit.

Foram relatados os ombros e a

coluna lombar os locais mais acometido e as lesões mais comum foram lesões musculares e dores nas articulações, sendo relatados como fatores de risco a carga de treinamento e as lesões anteriores a prática do CrossFit.
Embora o CrossFit possua um amplo número de participantes, a literatura ainda é limitada, mostrando os resultados positivos e benefícios. Com relação ao objetivo do presente estudo, os achados da investigação relacionadas à prevalência de lesão no CrossFit mostra maior predomínio de lesão no ombro e lombar entre os estudos analisados. Além disto, podemos afirmar que o índice ou proporção de lesões, de acordo como mostrado pela maioria dos estudos incluídos, deve levar em consideração uma grande variedade de fatores como lesões prévias, o protocolo aplicado, a presença de técnicos qualificados e outros.

O questionário aplicado aos praticantes de CrossFit no estudo verificou uma incidência de 3,24 por 1.000 horas de exercícios e a prevalência de $36 \%$ de lesões. As taxas desse estudo são parecidas com a de outras modalidades, como levantamento de peso básico, ginástica olímpica e Overhead Walking Lunge (OWL). Esse resultado sugere que os movimentos do corpo durante o treinamento é o principal determinante para as lesões. Com relação à localização das lesões, o ombro e coluna lombar baixa foram as regiões mais afetadas, devido serem regiões mais utilizadas no OWL e ginástica olímpica. Dessa forma os atletas precisam reduzir as execuções incorretas dos movimentos para menor risco de lesões. A associação entre lesão e o período de prática no CrossFit, mostra que atletas com maior tempo obtiveram 82,2\% superior a atletas menos experientes. Por isso, atletas em níveis competitivos são mais susceptíveis a sofrer mais lesão do que aqueles que são iniciantes e recreativos, devido ao período maior de exposição aos treinamentos, superação de limites e ao alto nível de habilidade técnica. ${ }^{2}$

No estudo realizado, notaram que, dos 3049 praticantes de CrossFit que participaram do questionário, $30,5 \%$ relataram sofrer uma lesão que estava relacionada à prática do esporte, não havendo uma diferença significativa entre os sexos. $60,4 \%$ da população lesionada relataram a lesão em uma única parte do corpo e 37,6\% relataram lesões em várias partes do corpo, estando prevalentes as lesões nos ombros (39\%), costas (36\%), joelhos (15\%), cotovelos (12\%) e pulsos (11\%). ${ }^{11}$

Foi empregado uma investigação epidemiológica descritiva utilizando um questionário eletrônico para verificar o risco de lesão em 252 participantes. Os resultados encontraram $56,1 \%$ dos participantes sofreram pelo menos uma lesão. Os locais mais comuns foram ombro, parte inferior das costas e seguido de joelho. Um total de $58,7 \%$ das lesões foi relatado como crônicas e uso excessivo. Os possíveis danos podem ser causados por: pouco tempo de participação no CrossFit associado a dimensões incorretas de exercícios, complexidade dos movimentos dos treinos, fadiga e peso exagerado. ${ }^{1}$

Segundo a pesquisa epidemiológica, a prevalência de lesões durante a prática de CrossFit é de 30\%. A respeito da taxa de lesões, esse estudo mostrou 1,34 por 1000 horas de treinamento. Considerando essas taxas apropriadas aos componentes, como sessão de treinamento, intensidade e período de recuperação, assim como os exercícios prescritos. Acerca dos tipos mais prevalentes de lesão, o estudo mostrou lesões articulares $(30,8 \%)$ e lesões musculares $(23,1 \%)$. Os locais de lesão mais frequente foram: ombro, coluna lombar e joelho, devido serem as regiões mais usadas nos exercícios básicos de levantamento de peso, levantamento olímpico e movimento de ginástica. Além disso, o estudo revelou que praticantes que treinam pouco, com menos experiência de treinamento ou que não participam de competições desenvolvem maior probabilidade de desenvolver lesões devido aos níveis baixos de condicionamento e técnica com execução incorreta. ${ }^{9}$

Na pesquisa epidemiológica, foi observada uma frequência de lesões nos ombros, joelhos e 
Revista de Ensino, Ciência e Inovação em Saúde v.2, n.3 (2021) 19-25

ISSN: 2675-9683/DOI: $10.51909 /$ recis.v2i3.166

parte inferior das costas, obtendo uma maior predisposição para lesão os atletas competidores ou que possuíam alto volume de treino, pela maior exposição aos movimentos. ${ }^{10}$

Na pesquisa, foi notável que praticantes do sexo masculino com menor frequência semanal de treino e menor experiência $(<1$ anos) foi mais propenso a relatar uma lesão em comparação com o sexo feminino. E os praticantes que tinham um volume maior de treino e uma experiência, justifica-se essa diferença com problemas de força ou de flexibilidade em praticantes menos experientes, impedindo dos mesmos completar alguns exercícios básicos, porém concluiu que o CrossFit é uma atividade segura quando realizada a mais de um ano, com um alto volume de treino semanal. ${ }^{12}$

Nos estudos, não são encontradas diferenças na associação entre lesões durante a prática do CrossFit com a idade, sexo, peso corporal, altura do atleta e prática de outros esportes. Porém, os estudos divergem em relação à associação da lesão e o tempo de prática. Em três estudos desta pesquisa, foi mostrado que pessoas que tinham mais tempo de prática eram mais propensos a desenvolver lesões em comparação com os que tinham um menor tempo de experiência, tendo uma maior probabilidade de lesão os atletas de nível competitivo. Por outro lado, nos outros três estudos incluídos foi observado que o menor tempo de experiência foi relacionado com um maior risco para lesões, uma vez que iniciantes devem ter o treino concentrado mais nos padrões de movimentos, com uma boa qualidade de treinamento, corrigindo o volume, complexidade e intensidade de acordo com o praticante, diminuindo o risco de lesões devido ao uso excessivo e à complexidade dos exercícios e movimentos, sendo a maioria das lesões de origem crônica/excessiva. ${ }^{1,2,9,10,11,12}$

\section{CONCLUSÃO}

De acordo com a literatura, conclui-se que o CrossFit parece ser uma modalidade segura, desde que realizada da forma adequada para cada indivíduo e com acompanhamento do profissional responsável, principalmente os praticantes iniciantes e os do nível de competição, que utilizam altas cargas e volume nos treinos, sendo um esporte que interfere em muitas variáveis do corpo humano e melhora a saúde e qualidade de vida. Porém, ainda são necessários mais estudos sobre o tema, pois existem incertezas na literatura consequentes de uma pouca quantidade de estudos com alto teor metodológico.

\section{REFERÊNCIAS BIBLIOGRÁFICAS}

1. Mehrab M, de Vos RJ, Kraan GA, Mathijssen NMC. Injury Incidence and Patterns Among Dutch CrossFit Athletes. Orthop J Sports Med. 2017; $5(12)$. https://doi.org/10.1177/2325967117745263

2. da Costa TS, Louzada CTN, Miyashita GK, da Silva PHJ, Sungaila HYF, Lara PHS, et al. CrossFit@: Injury prevalence and main risk factors. Clinics (Sao Paulo). 2019; 74:e1402. https://doi.org/10.6061/clinics/2019/e1402

3. Timón R, Olcina G, Camacho-Cardeñosa M, Camacho-Cardenosa A, Martinez-Guardado I, Marcos-Serrano M. 48-hour recovery of biochemical parameters and physical performance after two modalities of CrossFit workouts. Biol Sport. 2019; 36(3):283-289. https://doi.org/10.5114/biolsport.2019.85458

4. Hak PT, Hodzovic E, Hickey B. The nature and prevalence of injury during CrossFit training [published online ahead of print, 2013 Nov 22]. J StrengthCond Res. 2013; https://doi.org/10.1519/JSC.00000000000003 $\underline{18}$

5. Lopes P, Bezerra FHG, Filho NA, Brasileiro I Neto PP, Júnior FS. Lesões osteomioarticulares entre os praticantes de crossfit.

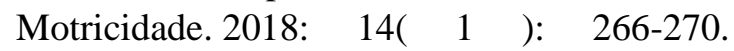
Disponível em: http://www.scielo.mec.pt/scielo.php?script=sc i arttext\&pid=S1646107X2018000100038\&1 $\underline{n g}=\mathrm{pt}$

6. Weisenthal BM, Beck CA, Maloney MD, DeHaven KE, Giordano BD. Injury Rate and Patterns Among CrossFit Athletes. Orthop J Sports Med. 2014;2(4). https://doi.org/10.1177/2325967114531177

7. Xavier AA, Lopes AMC. Lesões musculoesqueléticas em praticantes de crossfit [monografia]. Revista Interdisciplinar Ciências Médicas - MG 2017, 1(1): 11-27.

8. Drum SN, Bellovary BN, Jensen RL, Moore MT, Donath L. Perceived demands and postexercise physical dysfunction in CrossFit ${ }^{\circledR}$ compared to an ACSM based training session. J Sports Med Phys Fitness. 2017;57(5):604-609. https://doi.org/10.23736/S00224707.16.06243-5

9. Minghelli B, Vicente P. Musculoskeletal injuries in Portuguese CrossFit practitioners. J 
Revista de Ensino, Ciência e Inovação em Saúde v.2, n.3 (2021) 19-25

ISSN: 2675-9683/DOI: $10.51909 /$ recis.v2i3.166

Sports MedPhys Fitness. 2019;59(7):1213$1220 . \quad$ https://doi.org/10.23736/S00224707.19.09367-8

10. Montalvo AM, Shaefer H, Rodriguez B, Li T, Epnere K, Myer GD. Retrospective Injury Epidemiology and Risk Factors for Injury in CrossFit. J Sports Sci Med. 2017;16(1):53-59.

11. Feito Y, Burrows EK, Tabb LP. A 4-Year Analysis of the Incidence of Injuries Among
CrossFit-Trained Participants. Orthop J Sports Med. 2018;6(10). https://doi.org/10.1177/2325967118803100

12. Szeles PRQ, da Costa TS, da Cunha RA, Hespanhol L, Pochini AC, Ramos LA, et al. CrossFit and the Epidemiology of Musculoskeletal Injuries: A Prospective 12Week Cohort Study. Orthop J Sports Med. 2020;8(3).

https://doi.org/10.1177/2325967120908884 